\title{
$\beta$-Sitosterol Shows Potential to Protect Against the Development of High-Fructose Diet-Induced Metabolic Dysfunction in Female Rats
}

\author{
Nontobeko M. Gumede, Busisani W. Lembede, Richard L. Brooksbank, \\ Kennedy H. Erlwanger, and Eliton Chivandi
}

\author{
School of Physiology, Faculty of Health Sciences, University of the Witwatersrand, Johannesburg, South Africa.
}

\begin{abstract}
Metabolic syndrome (MetS) is a combination of risk factors that include insulin resistance, obesity, dyslipidemia, and hypertension. The consumption of high-fructose diets contributes to the development of MetS. $\beta$-sitosterol a naturally occurring phytosterol possesses antiobesogenic and antidiabetic effects. This study evaluated the potential protective effect of $\beta$-sitosterol against the development of metabolic dysfunction in growing female rats fed a high-fructose diet, mimicking children fed obesogenic diets. Thirty-five 21-day-old female Sprague Dawley rat pups were randomly allocated to and administered the following treatments: group 1-standard rat chow $(\mathrm{SRC})+$ plain drinking water $(\mathrm{PW})+$ plain gelatine cube (PC); group 2-SRC $+20 \% \mathrm{w} / \mathrm{w}$ fructose solution (FS) as drinking fluid $+\mathrm{PC}$; group 3-SRC $+\mathrm{FS}+100 \mathrm{mg} / \mathrm{kg}$ fenofibrate in gelatine cubes; group $4-\mathrm{SRC}+\mathrm{FS}+20 \mathrm{mg} / \mathrm{kg} \beta$-sitosterol gelatine cube (Bst); and group 5- $\mathrm{SRC}+\mathrm{PW}+\mathrm{Bst}$. Following 12 weeks of feeding, the rats were fasted overnight, weighed, and then euthanized. Plasma cholesterol, insulin, glucose, triglyceride, and adiponectin concentrations were determined. Visceral fat was dissected out and weighed. The highfructose diet increased $(P<.05)$ visceral adiposity and plasma triglyceride concentration but decreased $(P<.05)$ plasma adiponectin concentration. $\beta$-sitosterol prevented the high-fructose diet-induced visceral obesity, hypertriglyceridemia, and hypoadiponectinemia. $\beta$-sitosterol alone increased plasma adiponectin concentration and reduced plasma insulin concentration and homeostatic model assessment index. In conclusion, $\beta$-sitosterol could be potentially used to prevent highfructose diet-induced metabolic dysfunction.
\end{abstract}

\section{KEYWORDS: $\bullet \beta$-sitosterol $\bullet$ adiponectin $\bullet$ dyslipidemia $\bullet$ fructose $\bullet$ insulin resistance $\bullet$ metabolic syndrome}

\section{INTRODUCTION}

$\mathbf{T}$ he World Health Organization reported an increase in the prevalence of obesity among infants and young children (aged 0-5 years) from 32 million globally in 1990 to 41 million in 2016. ${ }^{1}$ If this trend continues globally, it is projected that the number of overweight or obese infants and young children will increase to 70 million by $2025 .{ }^{1}$ The prevalence of obesity is reported to be higher in female children compared to male children. ${ }^{2}$ Obesity is a major risk factor for the development of metabolic syndrome (MetS). ${ }^{3-5}$ MetS is defined as the concurrent manifestation of risk factors that increase the risk of developing type II diabetes mellitus (T-II-DM) and cardiovascular disease. ${ }^{6}$ These risk factors include insulin resistance, visceral adiposity, atherogenic dyslipidemia, and elevated blood pressure. ${ }^{7}$ Plasma triglyceride concentration and visceral obesity have been identified as accurate indicators for screening and predicting the risk for

Manuscript received 31 May 2019. Revision accepted 28 July 2019.

Address correspondence to: Nontobeko M. Gumede, School of Physiology, Faculty of Health Sciences, University of the Witwatersrand, 7 York Road, Parktown, Johannesburg, 2193, Republic of South Africa, E-mail: nontobeko.gumede@wits.ac.za
MetS and T-II-DM. ${ }^{8-10}$ Sedentary lifestyles and the consumption of obesogenic diets have been identified as the major causes of obesity and MetS. ${ }^{11}$

Fructose is used as a sweetening agent in various processed foods and beverages ${ }^{12}$ and due to poor dietary habits, there is an increase in the consumption of fructose-laden foods. The metabolism of fructose bypasses the phosphofructokinase rate-limiting step in hepatocytes and thus results in increased hepatic de novo lipogenesis (DNL). Increased lipogenesis contributes to the development of obesity and MetS. ${ }^{13-15}$

Some features of MetS can be treated or managed through the use of synthetic pharmaceutical agents such as fenofibrate, an antihyperlipidemic drug. ${ }^{16}$ However, synthetic pharmacological agents are costly and elicit deleterious side effects. ${ }^{17}$ This has resulted in a worldwide increase in the use of natural plant-derived ethnomedicines. ${ }^{16}$ Plant-derived ethnomedicines have been and continue to be used in disease management and their role in primary health care is expanding. ${ }^{16}$ Ethnomedicinal plants contain phytochemicals such as phytosterols that are known to have multiple biological benefits. ${ }^{16-18} \beta$-sitosterol is one of the most abundant and widely distributed phytosterols. ${ }^{19}$ It possesses antiobesogenic, anti-inflammatory, and antidiabetic properties. ${ }^{20,21} \beta$-sitosterol 
has been shown to increase the release of the antiinflammatory cytokine (interleukin-10) in macrophage cells. $^{22,23}$ Orally administered $\beta$-sitosterol [at 10,15 , and $20 \mathrm{mg} /(\mathrm{kg} \cdot \mathrm{d})$ ] for 21 days decreased glycated hemoglobin, serum glucose, and nitric oxide in streptozotocin-diabetic adult rats. ${ }^{20}$ These antidiabetic effects of $\beta$-sitosterol were attributed to its antioxidant activity and its ability to stimulate the regeneration of pancreatic $\beta$-cells. ${ }^{20}$ In a clinical study, the consumption of phytosterols was shown to be effective in treating hyper-low-density lipoprotein (LDL)-cholesterolemia in hypercholesterolemic patients. ${ }^{24}$ $\beta$-sitosterol exerted its antihyperlipidemic effects through the upregulation of cellular AMP-protein kinase (AMPK) activity, which suppresses DNL, increases fatty acid oxidation, and promotes adipose tissue mitochondrial integrity. ${ }^{25}$

Despite the evidence that obesity, MetS, and T-II-DM are increasing in children, the beneficial effects of $\beta$-sitosterol on metabolic health have been investigated mainly in adult animal models and adult human studies. Moreover, a large proportion of these studies have investigated the curative properties of $\beta$-sitosterol and not its prophylactic potential. Globally, it is well recognized that prevention of diseases reduces the burden of health care to a greater extent than the cure of diseases. Furthermore, most studies investigating diet-induced metabolic dysfunction have a tendency to use male animal models. ${ }^{26}$ However, the prevalence of obesity is reported to be higher in female children compared to male children. ${ }^{2}$ Thus, there is a need for studies that will interrogate the pathophysiology of diet-induced metabolic dysfunction and the potential plant-derived interventions initially using female and male animal experimental models before conducting human trials. This study, therefore, investigated the potential of orally administered $\beta$-sitosterol to prevent high-fructose diet-induced metabolic dysfunction in growing female rats mimicking children fed obesogenic diets.

\section{MATERIALS AND METHODS}

\section{Ethical approval}

Ethical clearance for the study was granted by the Animal Ethics Screening Committee (AESC) of the University of Witwatersrand (AESC clearance number 2017/08/55/B). This study was conducted in accordance with the internationally accepted principles and guidelines on the care and use of laboratory animals (National Research Council, 2010).

\section{Chemicals and reagents}

All the chemicals and reagents used in the study were of analytical grade. $\beta$-sitosterol, fenofibrate, and dimethyl sulfoxide (DMSO) were purchased from Sigma-Aldrich (St. Louis, MO, USA). The fructose was obtained from Nature's Choice (Randvaal, South Africa).

\section{Animals, housing and general care}

Thirty-five 21-day-old female Sprague Dawley rat pups were used in the experiment. Each rat was individually housed in a perspex cage with stainless steel mesh lids in the Central Animal Services, University of the Witwatersrand. The cages were lined with wood shavings for bedding. A 12$\mathrm{h}$ light and dark cycle was followed with lights on from 7 am to $7 \mathrm{pm}$. Room temperature was maintained at $26 \pm 2{ }^{\circ} \mathrm{C}$. The rats had ad libitum access to a standard rat chow (SRC) [Nutritionhub (PTY) Ltd., Stellenbosch, South Africa] and drinking fluid [water or $20 \%$ fructose solution (FS) as described below] throughout the experiment. The rat chow had the following nutrition content: calcium $14 \mathrm{~g} / \mathrm{kg}$, phosphorus $8 \mathrm{~g} / \mathrm{kg}$, vitamin $\mathrm{A}_{(\mathrm{min})} 16,000 \mathrm{IU} / \mathrm{kg}$, vitamin D $2000 \mathrm{IU} / \mathrm{kg}$ and vitamin E $100 \mathrm{mg} / \mathrm{kg}$, crude protein $240 \mathrm{~g} / \mathrm{kg}$, crude oils and fats $53 \mathrm{~g} / \mathrm{kg}$, linoleic acid $14 \mathrm{~g} / \mathrm{kg}$, crude fiber $45 \mathrm{~g} / \mathrm{kg}$, and crude ash $75 \mathrm{~g} / \mathrm{kg}$. The rat pups were acclimatized to handling for 2 days [postnatal days (PNDs) 21 and 22] before the commencement of the experiment on PND 23.

\section{Experimental design}

On PND 23 the 35 pups were randomly distributed and fed the following treatment regimens for 12 weeks:

group 1, the negative control $(n=7)-\mathrm{SRC}+$ plain drinking water $(\mathrm{PW})+$ plain gelatine cube $(\mathrm{PC})$,

group 2 in which metabolic dysfunction was induced $(n=7)-\mathrm{SRC}+20 \% \mathrm{w} / \mathrm{w}$ FS to drink $+\mathrm{PC}$,

group 3 in which fenofibrate was used a positive control for the prevention of metabolic dysfunction $(n=7)$ $\mathrm{SRC}+\mathrm{FS}+100 \mathrm{mg} / \mathrm{kg}$ body mass per day of fenofibrate in a gelatine cube $(\mathrm{FF})$,

group 4 in which the potential prophylactic effects of $\beta$-sitosterol against high-fructose diet-induced metabolic dysfunction were explored $(n=7)-\mathrm{SRC}+\mathrm{FS}+20 \mathrm{mg} / \mathrm{kg}$ body mass per day of $\beta$-sitosterol in a gelatine cube (Bst), and

group 5 in which the impact of $\beta$-sitosterol alone on the metabolic health of the rats was explored $(n=7)$ $\mathrm{SRC}+\mathrm{PW}+$ Bst.

The fenofibrate and $\beta$-sitosterol doses used were similar to those previously described by Hong et al. ${ }^{27}$ and Baskar et al. ${ }^{28}$ for nonalcoholic fatty liver disease and colon cancer respectively. Due to the poor aqueous solubility of $\beta$ sitosterol, DMSO $(0.5 \%)$ was used as a vehicle to dissolve $\beta$-sitosterol and the fenofibrate. ${ }^{28,29}$ The PCs in the control groups also contained $(0.5 \%)$ DMSO. The gelatine cubes were prepared as previously described by Kamerman et al. ${ }^{30}$ with modification. The gelatine cubes were prepared using $8 \mathrm{~g}$ brown sugar (Huletts, Tongaat, South Africa Ltd.) instead of $16 \mathrm{~g}$. Throughout the experiment, the rats were weighed twice weekly to ensure the maintenance of a constant dose of the interventions.

\section{Terminal procedures and sample collection}

Following 12 weeks of the administration of the treatments, the rats were fasted overnight but allowed access to PW. The rats were weighed and fasting blood glucose and triglyceride concentration was determined from a drop of blood obtained from the tail vein using a pinprick. ${ }^{31}$ Blood 
glucose and triglyceride concentration were measured using a calibrated glucose meter (Contour Plus Meter, Bayer, Isando-Johannesburg, South Africa) and a calibrated Accutrend triglyceride meter (Roche, Mannheim, Germany), respectively. Immediately thereafter the rats were euthanized with an intraperitoneal injection of sodium pentobarbitone at $200 \mathrm{mg} / \mathrm{kg}$ body mass. Following euthanasia, blood samples were collected, via cardiac puncture using $18 \mathrm{G}$ needles and $10 \mathrm{~mL}$ syringes, into heparinized blood tubes (Becton Dickinson Vacutainer Systems Europe, Meylan Cedex, France). The blood samples were centrifuged $20^{\circ} \mathrm{C}$ for $15 \mathrm{~min}$ at 5000 $g$ in a Sorvall RT ${ }^{\circledR}$ 6000B centrifuge (Pegasus Scientific, Inc., Rockville, MD, USA). The harvested plasma was stored in microtubes at $-20^{\circ} \mathrm{C}$ pending determination of plasma cholesterol, insulin, and adiponectin concentration.

The abdomen of each rat was opened via a midline incision and the visceral fat pad was dissected out and weighed. The visceral fat mass related to body mass (\% BM) was computed according to the formula: visceral fat mass (g)/ terminal body mass $(\mathrm{g}) \times 100$.

\section{Determination of adiponectin and insulin concentration}

Fasting adiponectin $(\mathrm{pg} / \mathrm{mL})$ and insulin $(\mathrm{ng} / \mathrm{mL})$ concentration were measured using an adiponectin and insulin enzyme-linked immunosorbent assay (ELISA) kit for rats (Elabscience Biotechnology Co, Wuhan, Hubei, China). A standard curve was constructed using calibrator concentrations. The concentrations of insulin and adiponectin in the samples were then determined from the constructed standard curve. Insulin resistance was estimated by computing the homeostatic model assessment index (HOMA-IR) using the fasting blood glucose and insulin concentration, by using the equation

HOMA-IR = insulin $(\mathrm{ng} / \mathrm{mL})$ blood glucose $(\mathrm{mmol} / \mathrm{L}) /$ $22.5 .^{32}$

\section{Determination of cholesterol}

The plasma cholesterol concentrations were determined using a colorimetric-based clinical chemistry analyzer (IDEXX VetTest ${ }^{\circledR}$ Clinical Chemistry Analyzer; IDEXX Laboratories, Inc., USA) as per the manufacturer's instructions.

\section{Statistical analysis}

GraphPad Prism 6.0 (Graph-pad Software, Inc., San Diego, CA, USA) software was used to analyze data. Data are expressed as mean \pm standard deviation. Data on weekly body mass were analyzed using a repeated measures analysis of variance (ANOVA). Multiple-group data were analyzed using one-way ANOVA, followed by Tukey's post hoc test. Significance was accepted when $P<.05$.

\section{RESULTS}

\section{Body mass}

Figure 1 shows the initial and terminal body masses of female rats across treatment regimens. The initial body masses of the rats were similar $(P>.05)$ (Fig. 1). Following the 12-week intervention across treatment regimens the rats had a significant increase $(P<.0001)$ in body mass (Fig. 1$)$. However, the terminal body masses were similar $(P>.05)$ across treatments (Fig. 1).

\section{Visceral obesity}

Figure 2 shows the absolute (A) and relative (B) visceral fat pad masses of female rats following respective treatments. Rats that were fed the high-fructose diet $(\mathrm{PC}+\mathrm{FS})$ had significantly $(P<.001)$ increased visceral fat mass (absolute and relative to body mass) compared to their control $(\mathrm{PC}+\mathrm{PW})$ counterparts (Fig. 2). $\beta$-sitosterol (Bst $+\mathrm{FS})$ prevented $(P<.001)$ the high-fructose diet-induced increase in absolute and relative (to body mass) visceral fat mass

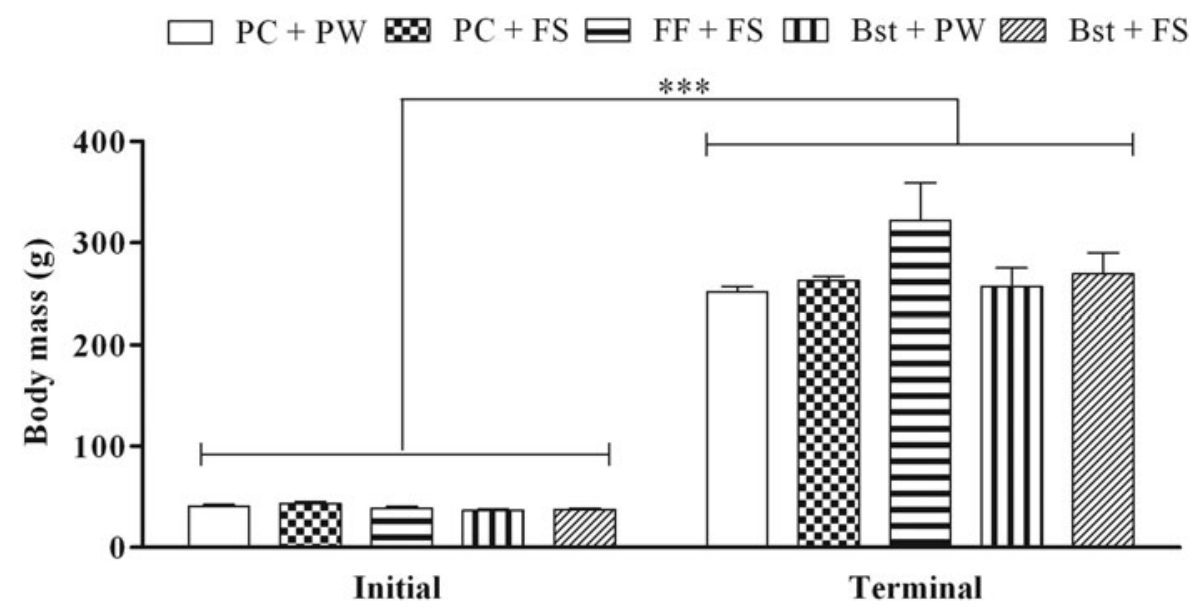

FIG. 1. Initial and terminal body masses of the female rats. Data presented as mean $\pm \mathrm{SD} ; n=7$ per treatment. $* * * P<.0001$; ANOVA, Tukey's post hoc test. ANOVA, analysis of variance; Bst $+\mathrm{FS}$, gelatine cube containing $\beta$-sitosterol at a dose of $20 \mathrm{mg} / \mathrm{kg}$ body mass per day $+20 \%$ (w/w) fructose solution to drink; Bst + PW, gelatine cube containing $\beta$-sitosterol at a dose of $20 \mathrm{mg} / \mathrm{kg}$ body mass per day + plain tap water to drink; FF + FS, gelatine cube containing fenofibrate at a dose of $100 \mathrm{mg} / \mathrm{kg}$ body mass per day $+20 \%(\mathrm{w} / \mathrm{w})$ fructose solution to drink; PC + FS, plain gelatine cubes $+20 \%(\mathrm{w} / \mathrm{w})$ fructose solution to drink; PC $+\mathrm{PW}$, plain gelatine cubes + plain tap water to drink; SD, standard deviation. 


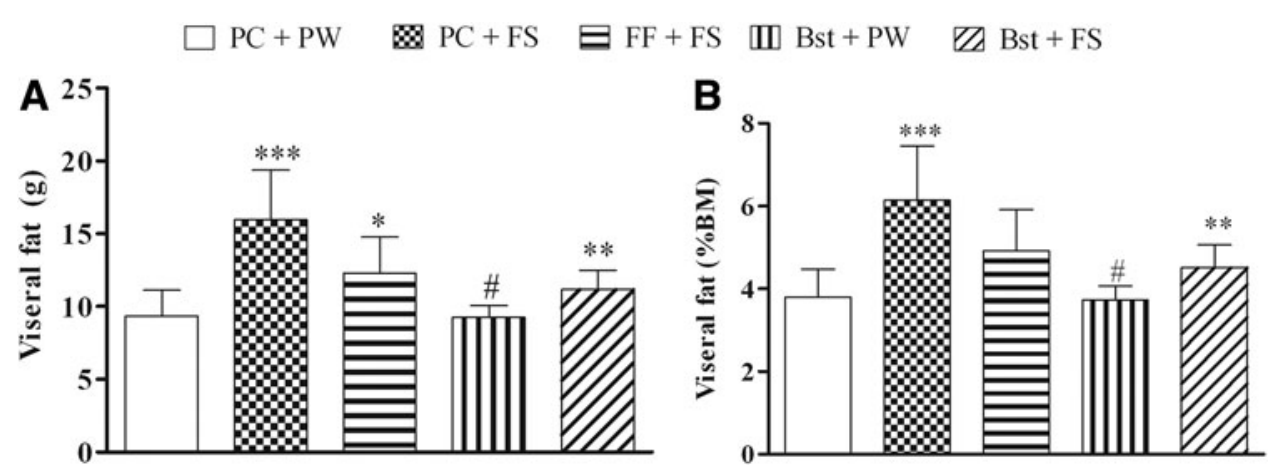

FIG. 2. Effect of $\beta$-sitosterol on absolute (A) and relative (B) visceral fat pad mass of female rats fed a high fructose diet. Data presented as mean $\pm \mathrm{SD} ; n=7$ per treatment. Absolute and relative visceral fat pad mass ANOVA $P<.0001 . * P<.05$ versus PC $+\mathrm{FS} ; * * P<.001$ versus PC + FS; $* * * P<.0001$ versus PC $+\mathrm{PW} ;{ }^{\#} P<.0001$ versus PC + FS; ANOVA, Tukey's multiple comparisons post hoc test. BM, body mass.

(Fig. 2). Rats fed fenofibrate (FF + FS) had significantly lower $(P<.05)$ absolute visceral fat mass compared to those on fructose alone (PC + FS) (Fig. 2).

\section{Triglyceride and cholesterol concentration}

Figure 3 shows the plasma triglyceride (A) and cholesterol (B) concentration in the female rats following their respective treatments. Rats that were fed high-fructose diet alone $(\mathrm{PC}+\mathrm{FS})$ had significantly increased $(P<.0001)$ plasma triglyceride concentration when compared to their control (PC $+\mathrm{PW}$ ) counterparts (Fig. 3). $\beta$-sitosterol (Bst + FS) prevented $(P<.0001)$ the high-fructose diet-induced hypertriglyceridemia (Fig. 3). Fenofibrate (FF + FS) failed to prevent $(P>.05)$ the high-fructose diet-induced hypertriglyceridemia (Fig. 3). Although the high-fructose diet had not significantly increased $(P>.05)$ the plasma total cholesterol concentration (Fig. 3), the high-fructose diet-fed rats had 26\% higher $(P=.08)$ plasma total cholesterol compared to that of rats fed the control diet. Rats fed the high-fructose diet in combination with fenofibrate (FF $+\mathrm{FS})$ or $\beta$-sitosterol $($ Bst + FS) had significantly higher $(P<.05)$ plasma cholesterol concentration compared to their control $(\mathrm{PC}+\mathrm{PW})$ counterparts (Fig. 3). Rats that fed $\beta$-sitosterol alone (Bst + PW) had significantly lower $(P<.001)$ plasma cholesterol concentration compared to their counterparts who fed the high-fructose diet (PC + FS; Bst + FS; FF + FS) (Fig. 3) .

\section{Glucose, insulin, adiponectin, and HOMA-IR}

Figure 4 shows the glucose (A), insulin (B), and adiponectin (C) concentration and HOMA-IR (D) of the female rats following their respective treatments. The high-fructose diet had no effect on glucose and insulin concentration and HOMA-IR (Fig. 4). Rats fed the high-fructose diet (PC + FS) had an undetectable adiponectin concentration (Fig. 4), thus were assigned the lowest detectable ELISA adiponectin concentration of $46.88 \mathrm{pg} / \mathrm{mL}$. Rats fed the high-fructose diet in combination with $\beta$-sitosterol (Bst $+\mathrm{FS}$ ) or fenofibrate $(\mathrm{FF}+\mathrm{FS})$ had significantly higher $(P<.0001)$ plasma adiponectin concentration compared to their control $(\mathrm{PC}+\mathrm{PW})$ and high-fructose diet (PC + FS) counterparts (Fig. 4). $\beta$ sitosterol alone $(\mathrm{Bst}+\mathrm{PW})$ significantly increased $(P<.001$ compared to $\mathrm{PC}+\mathrm{PW}$ and $\mathrm{PC}+\mathrm{FS})$ plasma adiponectin concentration and lowered $(P<.001$ compared to all other treatments) plasma insulin concentration and HOMA-IR (Fig. 4).

\section{DISCUSSION}

The study aimed to investigate the potential of orally administrated $\beta$-sitosterol to protect growing female rats against the development of fructose-induced metabolic dysfunction. Results from the current study showed that the high-fructose diet had no effect on body mass gain of female

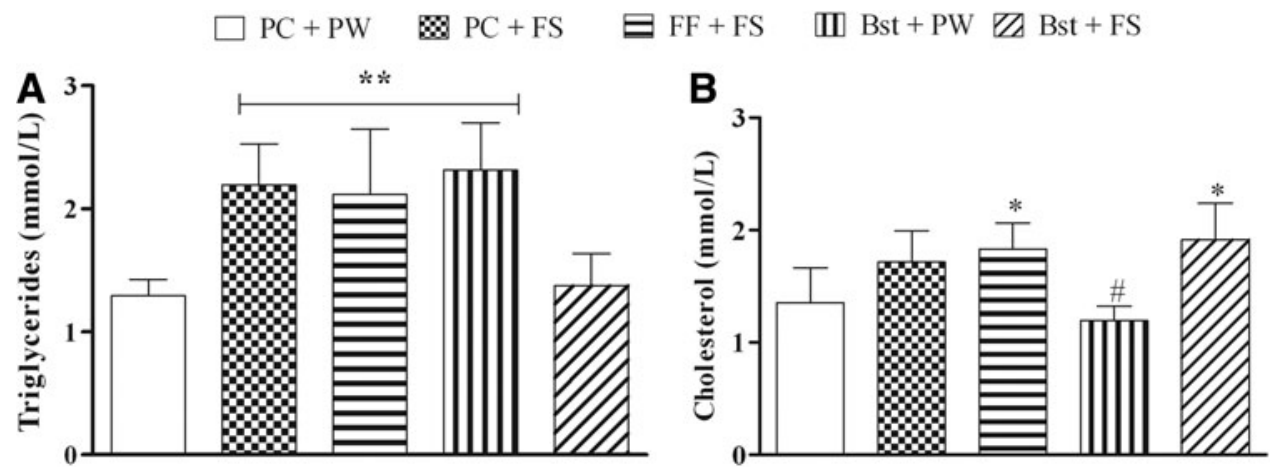

FIG. 3. Effect of $\beta$-sitosterol on plasma triglyceride (A) and cholesterol (B) concentration of female rats fed a high-fructose diet. Data presented as mean $\pm \mathrm{SD} ; n=7$ per treatment. Triglyceride and cholesterol ANOVA $P<.0001 . * P<.05$ versus $\mathrm{PC}+\mathrm{PW} * * * P<.001$ versus $\mathrm{PC}+\mathrm{PW}$ and $\mathrm{BS}$ $+\mathrm{FS} ;{ }^{\#} P<.05$ versus PC + FS, FF + FS and Bst + FS; ANOVA, Tukey's multiple comparisons post hoc test. 

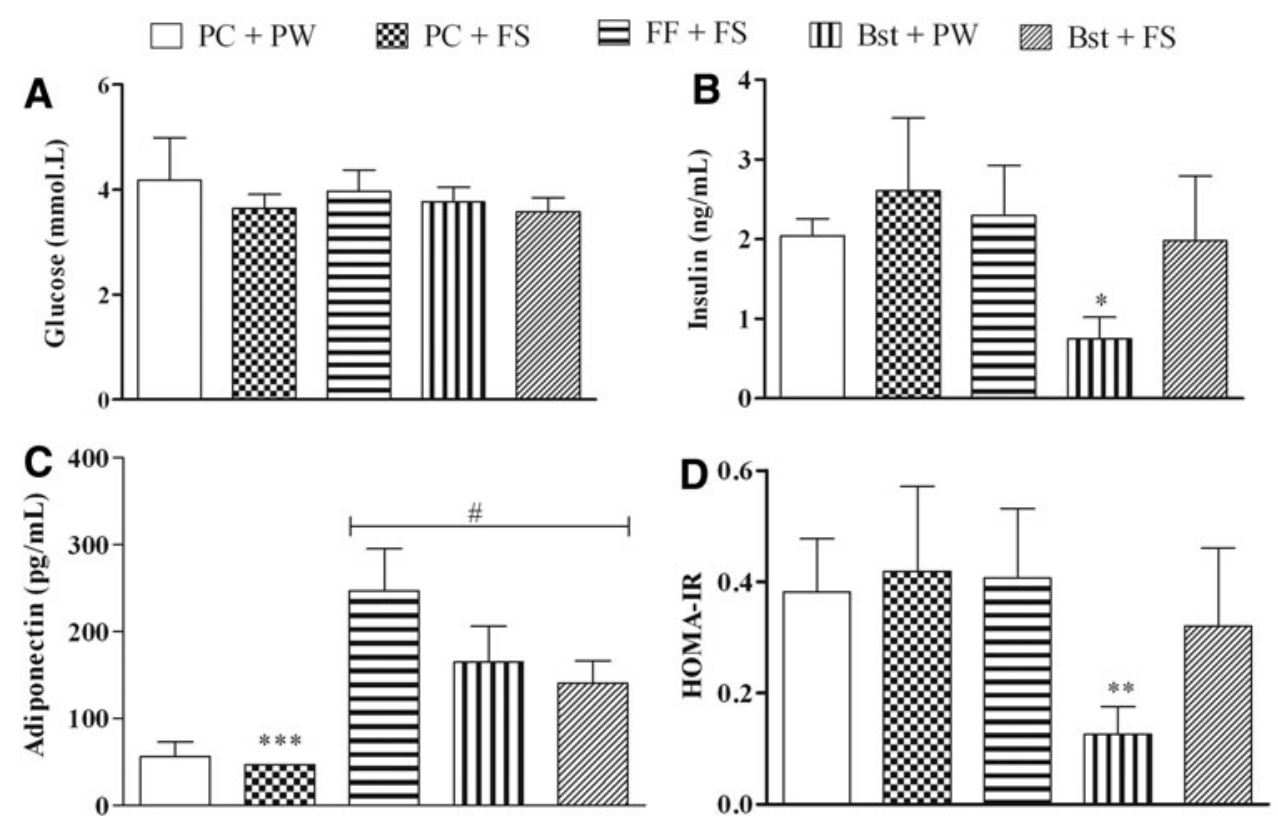

FIG. 4. Effect of $\beta$-sitosterol on glucose (A), insulin (B), and adiponectin (C) concentration and HOMA-IR (D) of high-fructose diet-fed rats. Data presented as mean $\pm \mathrm{SD} ; n=6-7$ per treatment. Blood glucose ANOVA $P=.1424$; insulin $P=.0004$; HOMA-IR $P=.0011$; adiponectin $P<.0001 . * P<.05$ when compared to all groups; $* * P<.001$ when compared to $\mathrm{PC}+\mathrm{PW}, \mathrm{PC}+\mathrm{FS}$, and FF $+\mathrm{FS} ; * * * P<.0001$ when compared to $\mathrm{FF}+\mathrm{FS}$, Bst + FS, and Bst + PW; ${ }^{\#} P<.0001$ when compared to PC + PW; ANOVA, Tukey's multiple comparisons post hoc test. HOMA-IR, homeostatic model assessment index.

rats (Fig. 1). These findings are in agreement with Ramos et al. who reported that the consumption of $20 \%$ fructose diet for 8 weeks had no effect of body mass gain but caused increased visceral adiposity. ${ }^{33}$ Ramos et al. contended that the observed increase in adiposity without a concomitant increase in body mass to be a consequence of the high adipogenic and low energetic nature of fructose. ${ }^{33} \mathrm{We}$ therefore speculate that the observed dichotomy between the body mass and visceral adiposity in the current study is a result of the low energy efficiency of fructose. $\beta$-sitosterol and fenofibrate had no effect on body mass gain in the current study (Fig. 1). Therefore, these findings suggest that $\beta$-sitosterol and fenofibrate had no effect on body mass gain in the current study (Fig. 1). Body mass gain is an important indirect indicator of an animal's health status and therefore, these findings suggest that $\beta$-sitosterol and fenofibrate did not negatively impact the rats' health as indicated by body mass. In the present study, the consumption of a high-fructose diet for 12 weeks by female rats resulted in metabolic dysfunction characterized by visceral obesity, hypoadiponectinemia, and hypertriglyceridemia. $\beta$-sitosterol protected the female rats against the development of highfructose diet-induced metabolic dysfunction. Fenofibrate only prevented the high-fructose diet-induced visceral obesity and hypoadiponectinemia.

In the liver, the metabolism of each molecule of fructose produces three carbon intermediates, which can enter the glycolytic pathway. ${ }^{34}$ These intermediates may be interconverted or further metabolised to acetyl-CoA. ${ }^{35}$ AcetylCoA can be converted to malonyl-CoA, a metabolite that is utilized in de novo triglyceride synthesis. ${ }^{36}$ The acetyl-CoA can also form HMG-CoA and undergo a series of reactions to form cholesterol ${ }^{37}$ The newly synthesized triglycerides are packed into very low-LDL and transported to the peripheral adipose stores and thus causing dyslipidemia and visceral obesity ${ }^{38,39}$ In the current study, the high-fructose diet caused hypertriglyceridemia and visceral adiposity (Figs. 2 and 3). These findings suggest that the high-fructose diet caused de novo triglyceride synthesis ${ }^{40}$ that resulted in hypertriglyceridemia and visceral adiposity. ${ }^{41}$ Several studies have reported that MetS can be accurately diagnosed using only plasma triglyceride concentration and visceral obesity. ${ }^{9,10}$ Thus, hypertriglyceridemia and visceral obesity are key predictors of MetS. In the current study, consumption of the high-fructose diet increased $(P>.05)$ mean plasma cholesterol by $27 \%$ when compared to control (Fig. 3). It has been reported that a $10 \%$ change in total cholesterol concentration is clinically relevant. ${ }^{42}$ A $10 \%$ increase in cholesterol significantly increases the risk of coronary heart disease-related mortality. ${ }^{42}$ Thus, findings of the current study suggest that the highfructose diet might have hypercholesterolemic effects.

In the present study, $\beta$-sitosterol prevented the high-fructose diet-induced hypertriglyceridemia and visceral obesity (Figs. 2 and 3). These antiobesogenic effects of $\beta$-sitosterol might be attributed to its ability to decrease hepatic DNL through AMPK activation. ${ }^{25}$ In the current study, cholesterol increased when $\beta$-sitosterol was administered to high-fructose diet-fed rats (Fig. 3). However, when $\beta$-sitosterol was administrated alone it resulted in a $12 \%$ decrease in mean plasma cholesterol concentration compared to control. In vitro, $\beta$-sitosterol was reported to decrease cholesterol synthesis by decreasing HMG-CoA reductase activity. ${ }^{43}$ Thus, it could be speculated 
that in this study the administration of $\beta$-sitosterol exacerbated the hypercholesterolemic effects of the high-fructose diet. A possible explanation could be that $\beta$-sitosterol and fructose, together, have synergistic effects that consequently resulted in the hypercholesterolemia. At the moment the mechanisms involved in these findings are unknown and thus further investigation are needed.

Fenofibrate, an antihyperlipidemic drug, was used as a positive control in the current study. It exerts antiobesogenic effects by stimulating peroxisome proliferator-activated receptor $\alpha(\mathrm{PPAR}-\alpha)$-mediated lipolysis and fatty acid $\beta$ oxidation in adipose tissues. ${ }^{44}$ PPAR- $\alpha$ activates genes that regulate lipoprotein lipase, carnitine palmitoyltransferase, and acyl-Co-A oxidase activity in the liver and in adipocytes. ${ }^{45,46}$ Lipoprotein lipase hydrolyses triglycerides into fatty acid and glycerol. ${ }^{47,48}$ Carnitine palmitoyltransferase facilitates free fatty acids uptake into the mitochondria. Inside the mitochondria, acyl-Co-A oxidase promotes fatty acid $\beta$-oxidation ${ }^{48}$ In the current study, fenofibrate prevented the high-fructose diet-induced visceral obesity (Fig. 2). This finding is in agreement with Jeong and Yoon who reported that fenofibrate decreased visceral fat mass in high fat diet-fed obese mice. ${ }^{44}$ It could be speculated that the decrease in visceral fat mass in the current study following fenofibrate administration was due to upregulated triglyceride hydrolysis and fatty acid $\beta$-oxidation. In contrast to previous studies, fenofibrate failed to prevent the high-fructose dietinduced hypertriglyceridemia and hypercholesterolemia in the current study. Fructose causes dyslipidemia by increasing DNL in the liver. ${ }^{40}$ Thus, it could be speculated that fenofibrate was unable to prevent the high-fructose diet-induced DNL in the liver.

Several studies have reported that visceral adiposity is associated with decreased adiponectin concentration. ${ }^{49,50}$ Visceral adiposity increases the production of tumor necrosis factor- $\alpha,{ }^{51}$ which in turn inhibits adiponectin gene expression. ${ }^{52}$ Adiponectin, an anti-inflammatory adipokine, increases insulin sensitivity by stimulating the activity of PPAR- $\alpha$ and AMPK ${ }^{53}$ In the present study, rats fed a highfructose diet showed undetectable adiponectin concentration (Fig. 4). This finding suggests that the high-fructose diet exerted hypoadiponectinemia effects (Fig. 4). The highfructose diet might have decreased adiponectin by causing visceral adiposity. ${ }^{41}$ HOMA-IR is used to assess insulin resistance. ${ }^{54}$ Although the high-fructose diet decreased adiponectin, it had no effect on insulin concentration and HOMA-IR (Fig. 4).

In the current study, the oral administration of $\beta$-sitosterol alone or with the high-fructose diet resulted in increased plasma adiponectin concentration (Fig. 4). $\beta$-sitosterol may have exerted its hyperadiponectinemia effects through the reduction of visceral adiposity. Collins et al. reported that the hyperadiponectinemia effect of plant sterols is attributed to their anti-inflammatory activities. ${ }^{55}$ The administration of $\beta$-sitosterol alone significantly decreased plasma insulin concentration and increased insulin sensitivity (Fig. 4). These results are in tandem with those by Radika et al. who reported reduced insulin concentration and improved insulin sensitivity following 4 weeks administration of $\beta$-sitosterol to adult Wistar rats fed a high-fat diet. ${ }^{56} \beta$-sitosterol reduced plasma insulin concentration and HOMA-IR by exerting anti-inflammatory effects and by decreasing the expression of inducible nitric oxide synthase (iNOS) that interferes with insulin signaling. ${ }^{57}$ Therefore, it could be speculated that $\beta$ sitosterol decreased plasma insulin concentration and increased insulin sensitivity through anti-inflammatory mechanisms and suppressing iNOS-induced insulin signaling interference. The oral administration of fenofibrate significantly increased plasma adiponectin concentration but had no effect on insulin concentration and HOMA-IR (Fig. 4); thus, it can be speculated that fenofibrate increased adiponectin concentration by decreasing visceral adiposity.

\section{CONCLUSION}

We have shown that feeding a high-fructose diet to growing female rats for 12 weeks caused metabolic dysfunction characterized by visceral adiposity, hypertriglyceridemia, and hypoadiponectinemia. $\beta$-sitosterol protected the growing female rats against the development of high-fructose dietinduced metabolic dysfunction. In conclusion, $\beta$-sitosterol could potentially be exploited as a prophylactic agent against diet-induced metabolic dysfunction in children.

\section{ACKNOWLEDGMENTS}

We thankfully acknowledge the Faculty of Health Sciences Research Committee of the University of the Witwatersrand, Johannesburg and the National Research Foundation of South Africa for funding the research. We also wish to acknowledge the staff of the University of the Witwatersrand Central Animal Service unit for assistance with the care and welfare of the rats. The authors also wish to acknowledge Ms. Monica Gomez for her technical input.

\section{AUTHOR DISCLOSURE STATEMENT}

The authors have no conflicts of interest to declare.

\section{FUNDING INFORMATION}

The authors acknowledge the Faculty of Health Sciences Research Committee of the University of the Witwatersrand, Johannesburg and, the National Research foundation of South Africa (Grant number: PR_IFR190125410875).

\section{REFERENCES}

1. World Health Organisation: Global Report Status on Noncommunicable Diseases 2014. WHO, Geneva, Switzerland, 2014.

2. Maruf FA, Aronu U, Chukwuegbu K, Aronu AE: Influence of gender on prevalence of overweight and obesity in Nigerian schoolchildren and adolescents. Tanzan J Health Res 2013;15:1-6.

3. Alberti KG, Zimmet P, Shaw J, Alberti KGM, Zimmet P, Shaw J: The metabolic syndrome-A new worldwide definition. Lancet 2005;366:1059-1062. 
4. Grundy SM, Cleeman JI, Daniels SR, et al.: Diagnosis and management of the metabolic syndrome. An American Heart Association/National Heart, Lung, and Blood Institute Scientific Statement. Executive summary. Cardiol Rev 2005;13:2735-2752.

5. Nolan PB, Carrick-Ranson G, Stinear JW, Reading SA, Dalleck LC: Prevalence of metabolic syndrome and metabolic syndrome components in young adults: A pooled analysis. Prev Med Reports 2017;7:211-215.

6. Peeters CFW, Dziura J, van Wesel F, Wesel FVAN. Pathophysiological domains underlying the metabolic syndrome: An alternative factor analytic strategy. Ann Epidemiol 2014;24:1-17.

7. Fiszman M, Rosemblat G, Ahlers CB, Rindflesch TC: Identifying risk factors for metabolic syndrome in biomedical text. AMIA Annu Symp Proc 2007;2007:249-253.

8. Li Z, Deng ML, Tseng C-H, Heber D: Hypertriglyceridemia is a practical biomarker of metabolic syndrome in individuals with abdominal obesity. Metab Syndr Relat Disord 2012;11:87-91.

9. He S, Zheng Y, Shu Y, He J, Wang Y, Chen X: Hypertriglyceridemic waist might be an alternative to metabolic syndrome for predicting future diabetes mellitus. PLoS One 2013;8:e73292.

10. Ren Y, Liu Y, Sun X, et al.: Hypertriglyceridemia-waist and risk of developing type 2 diabetes: The Rural Chinese Cohort Study. Sci Rep 2017;7:1-8.

11. Bentov Y: "A Western diet side story": The effects of transitioning to a Western-type diet on fertility. Endocrinology 2014; 155:2341-2342.

12. Zargaraan A, Kamaliroosta L, Yaghoubi AS, Mirmoghtadaie L: Effect of substitution of sugar by high fructose corn syrup on the physicochemical properties of bakery and dairy products: A review. Nutr Food Sci Res 2016;3:3-11.

13. Bray GA, Nielsen SJ, Popkin BM: Consumption of high-fructose corn syrup in beverages may play a role in the epidemic of obesity. Am J Clin Nutr 2004;79:537-543.

14. Lozano I, Van Der Werf R, Bietiger W, et al.: High-fructose and high-fat diet-induced disorders in rats: Impact on diabetes risk, hepatic and vascular complications. Nutr Metab 2016;13:1-13.

15. Rebolledo O, Marra C, Raschia A, Rodriguez S, Gagliardino J: Abdominal adipose tissue: Early metabolic dysfunction associated to insulin resistance and oxidative stress induced by an unbalanced diet. Horm Metab Res 2008;40:794-800.

16. Valasek MA, Clarke SL, Repa JJ: Fenofibrate reduces intestinal cholesterol absorption via PPAR $\alpha$-dependent modulation of NPC1L1 expression in mouse. J Lipid Res 2007;48:2725-2735.

17. Florez H, Temprosa MG, Orchard TJ, et al.: Metabolic syndrome components and their response to lifestyle and metformin interventions are associated with differences in diabetes risk in persons with impaired glucose tolerance. Diabetes Obes Metab 2014;16:326-333.

18. Fyhrquist P, Mwasumbi L, Hæggström C-A, Vuorela H, Hiltunen $\mathrm{R}$, Vuorela P: Ethnobotanical and antimicrobial investigation on some species of Terminalia and Combretum (Combretaceae) growing in Tanzania. J Ethnopharmacol 2002;79:169-177.

19. Kim KS, Yang HJ, Lee JY, et al.: Effects of $\beta$-sitosterol derived from Artemisia capillaris on the activated human hepatic stellate cells and dimethylnitrosamine-induced mouse liver fibrosis. BMC Complement Altern Med 2014;14:1-10.

20. Gupta RS, Sharma AK, Dobhal MP, Sharma MC, Gupta RS: Antidiabetic and antioxidant potential of $\beta$-sitosterol in streptozotocininduced experimental hyperglycemia. J Diabetes 2011;3:29-37.
21. Vivancos $\mathbf{M}$, Moreno JJ: $\beta$-Sitosterol modulates antioxidant enzyme response in RAW 264.7 macrophages. Free Radic Biol Med 2005;39:91-97.

22. Gupta M, Nath R, Srivastava N, Shanker K, Kishor K, Bhargava $\mathrm{K}$ : Anti-inflammatory and antipyretic activities of $\beta$-sitosterol. Planta Med 1980;39:157-163.

23. Valerio M, Awad AB: $\beta$-sitosterol down-regulates some proinflammatory signal transduction pathways by increasing the activity of tyrosine phosphatase SHP-1 in J774A.1 murine macrophages. Int Immunopharmacol 2011;11:1012-1017.

24. Gylling H, Plat J, Turley S, et al:: Plant sterols and plant stanols in the management of dyslipidaemia and prevention of cardiovascular disease. Atherosclerosis 2014;232:346-360.

25. Hwang S-LL, Kim H-NN, Jung H-HH, et al:: Beneficial effects of $\beta$-sitosterol on glucose and lipid metabolism in L6 myotube cells are mediated by AMP-activated protein kinase. Biochem Biophys Res Commun 2008;377:1253-1258.

26. Wayhart JP, Lawson HA: Animal models of metabolic syndrome. Anim Model Study Hum Dis Second Ed 2017;13:221-243.

27. Hong XZ, Li L Da, Wu LM: Effects of fenofibrate and xuezhikang on high-fat diet-induced non-alcoholic fatty liver disease. Clin Exp Pharmacol Physiol 2007;34:27-35.

28. Baskar AA, Ignacimuthu S, Paulraj GM, Al Numair KS: Chemopreventive potential of beta-Sitosterol in experimental colon cancer model - an in vitro and in vivo study. BMC Complement Altern Med 2010;10:1-10.

29. McCormack S, Polyak E, Ostrovsky J, et al:: Pharmacologic targeting of sirtuin and PPAR signaling improves longevity and mitochondrial physiology in respiratory chain complex I mutant Caenorhabditis elegans. Mitochondrion 2015;22:45-59.

30. Kamerman P, Modisa B, Mphahlele N: Atorvastatin, a potent HMG-CoA reductase inhibitor, is not antipyretic in rats. $J$ Therm Biol 2004;29:431-435.

31. Ndong M, Uehara M, Katsumata S-I, Suzuki K: Effects of oral administration of Moringa oleifera Lam on glucose tolerance in Goto-Kakizaki and Wistar rats. J Clin Biochem Nutr 2007;40: 229-233.

32. Divi S, Bellamkhonda R, Dasireddy S: Evaluation of antidiabetic and antihyperlipedemic potential of aqueous extract of Moringa oleifera in fructose fed insulin resistant and STZ induced diabetic. Asian J Pharm Clin Res 2012;5:67-72.

33. Ramos VW, Batista LO, Albuquerque KT: Effects of fructose consumption on food intake and biochemical and body parameters in Wistar rats. Rev Port Cardiol 2017;36:937-941.

34. Khitan Z, Kim DH: Fructose: A key factor in the development of metabolic syndrome and hypertension. J Nutr Metab 2013; 2013:1-12.

35. Nomura K, Yamanouchi T: The role of fructose-enriched diets in mechanisms of nonalcoholic fatty liver disease. J Nutr Biochem 2012;23:203-208.

36. Solinas G, Borén J, Dulloo AG: De novo lipogenesis in metabolic homeostasis: More friend than foe? Mol Metab 2015;4:367-377.

37. Siperstein MD, Guest MJ: Studies on the site of the feedback control of cholesterol synthesis. J Clin Invest 1960;39:642-652.

38. Klop B, Elte JWF, Cabezas MC: Dyslipidemia in obesity: Mechanisms and potential targets. Nutrients 2013;5:1218-1240.

39. Rizkalla SW: Health implications of fructose consumption: A review of recent data. Nutr Metab (Lond) 2010;7:1-17. 
40. Welsh JA, Sharma A, Abramson JL, Vaccarino V, Gillespie C, Vos MB: Caloric sweetener consumption and dyslipidemia among US adults. J Am Med Assoc 2010;303:1490-1497.

41. Stanhope KL, Schwarz JM, Keim NL, et al. Consuming fructosesweetened, not glucose-sweetened, beverages increases visceral adiposity and lipids and decreases insulin sensitivity in overweight/obese humans. J Clin Invest 2009;119:1322-1334.

42. Gould AL, Davies GM, Alemao E, Yin DD, Cook JR: Cholesterol reduction yields clinical benefits: Meta-analysis including recent trials. Clin Ther 2007;29:778-794.

43. Field FJ, Born E, Mathur SN: Effect of micellar beta-sitosterol on cholesterol metabolism in CaCo-2 cells. J Lipid Res 1997;38: 348-360.

44. Jeong S, Yoon M. Fenofibrate inhibits adipocyte hypertrophy and insulin resistance by activating adipose PPAR $\alpha$ in high fat dietinduced obese mice. Exp Mol Med 2009;41:397-405.

45. Minnich A, Tian N, Byan L, Bilder G: A potent PPAR $\alpha$ agonist stimulates mitochondrial fatty acid $\beta$-oxidation in liver and skeletal muscle. Am J Physiol Metab 2001;280:E270-E279.

46. Schoonjans K, Peinado-Onsurbe J, Lefebvre AM, et al.: PPARalpha and PPARgamma activators direct a distinct tissue-specific transcriptional response via a PPRE in the lipoprotein lipase gene. EMBO J 1996;15:5336-5348.

47. Uchida A, Slipchenko MN, Cheng JX, Buhman KK: Fenofibrate, a peroxisome proliferator-activated receptor $\alpha$ agonist, alters triglyceride metabolism in enterocytes of mice. Biochim Biophys Acta 2011;1811:170-176.

48. Hashimoto T, Fujita $\mathrm{T}$, Usuda $\mathrm{N}$, et al.: Peroxisomal and mitochondrial fatty acid $\beta$-oxidation in mice nullizygous for both peroxisome proliferator-activated receptor and peroxisomal fatty acyl-CoA oxidase: Genotype correlation with fatty liver phenotype. J Biol Chem 1999;274:19228-19236.
49. Balsan GA, Vieira JL da C, Oliveira AM de, Portal VL: Relationship between adiponectin, obesity and insulin resistance. Rev Assoc Med Bras 2015;61:72-80.

50. Forny-Germano L, De Felice FG, Do Nascimento Vieira MN: The role of leptin and adiponectin in obesity-associated cognitive decline and Alzheimer's disease. Front Neurosci 2019; 13:1-19.

51. Hotamisligil GS, Arner P, Caro JF, Atkinson RL, Spiegelman BM: Increased adipose tissue expression of tumor necrosis factor- $\alpha$ in human obesity and insulin resistance. J Clin Invest 1995;95:2409-2415.

52. Maeda N, Takahashi M, Funahashi T, et al. PPAR $\gamma$ ligands increase expression and plasma concentrations of adiponectin, an adipose-derived protein. Diabetes 2001;50:2094-2099.

53. Kadowaki T, Yamauchi T: Adiponectin and adiponectin receptors. Endocr Rev 2005;26:439-451.

54. Matthews DR, Hosker JP, Rudenski AS, Naylor BA, Treacher DF, Turner RC: Homeostasis model assessment: Insulin resistance and beta-cell function from fasting plasma glucose and insulin concentrations in man. Diabetologia 1985;28:412-419.

55. Collins M, Varady KA, Jones PJH: Modulation of apolipoprotein $\mathrm{A} 1$ and $\mathrm{B}$, adiponectin, ghrelin, and growth hormone concentrations by plant sterols and exercise in previously sedentary humans. Can J Physiol Pharmacol 2007;85:903910.

56. Radika MK, Viswanathan P, Anuradha CV: Nitric oxide mediates the insulin sensitizing effects of $\beta$-sitosterol in high fat dietfed rats. Nitric Oxide Biol Chem 2013;32:43-53.

57. Fujimoto M, Shimizu N, Kunii K, Martyn JAJ, Ueki K, Kaneki M: A role of iNOS in the expression of IRS-1, IRS-2 and SREBP-1c in the liver of obese, diabetic mice. Diabetes 2005; 54:1340-1348. 